\title{
Cost-efficient Selective Network Caching in Large-Area Vehicular Networks using Multi-objective Heuristics
}

\author{
Miren Nekane Bilbao ${ }^{1}$, Cristina Perfecto ${ }^{1}$, and Javier Del Ser ${ }^{1,2,3}$ \\ 1 University of the Basque Country UPV/EHU, 48013 Bilbao, Spain \\ \{nekane.bilbao, cristina.perfecto, javier.delser\}@ehu.eus \\ 2 TECNALIA, E-48160 Derio, Spain \\ 3 Basque Center for Applied Mathematics (BCAM), 48009 Bilbao, Spain
}

\begin{abstract}
In the last decade the interest around network caching techniques has augmented notably for alleviating the ever-growing demand of resources by end users in mobile networks. This gained momentum stems from the fact that even though the overall volume of traffic retrieved from Internet has increased at an exponential pace over the last years, several studies have unveiled that a large fraction of this traffic is usually accessed by multiple end users at nearby locations, i.e. content demands are often local and redundant across terminals close to each other, even in mobility. In this context this manuscript explores the application of multi-objective heuristics to optimally allocate cache profiles over urban scenarios with mobile receivers (e.g. vehicles). To this end we formulate two conflicting objectives: the utility of the cache allocation strategy, which roughly depends on the traffic offloaded from the network and the number of users demanding contents; and its cost, given by an cost per unit of stored data and the rate demanded by the cached profile. Simulations are performed and discussed over a realistic vehicular scenario modeled over the city of Cologne (Germany), from which it is concluded that the proposed heuristic solver excels at finding caching solutions differently balancing the aforementioned objectives.
\end{abstract}

Keywords: Network caching; Vehicular networks; Heuristics

\section{Introduction}

Content caching and delivery leverage the storage capabilities and wireless interfaces of user terminals to store concurrently and redundantly accessed contents. These functionalities are applied on the realistic assumption that a large percentage of such contents will be requested by nearby users in the near future. By eliminating the need for retrieving such contents from the core network the traffic flow demand from the telecommunication operator can be contained, which is crucial in light of recent forecasts around Internet stats with annual network traffic volumes expected to score above the zettabyte in 2016 [1]. This strategy, 
which finds its roots in the early stages of Internet (with local HTTP caches and proxies becoming the de facto standard $[2,3]$ ), has reborn in the last years as a means to reduce network traffic, bottlenecks, and user access latencies in $5 \mathrm{G}$ networks [4-6]. In-network caching, which refers to the deployment of contentdriven caches through a given network, is one of the mechanisms that lie at the core of the Information-centric networking (ICN) paradigm, which aims at changing the Internet infrastructure from its traditional host-centric, connectivity based principle [7]. ICN envisages a network composed by named information entities (i.e. contents) whose labels are irrelevant with respect to their location over the network infrastructure. This radical swift involves that users consume contents rather than resources, hence information entities embody the resource unit around which users' network operations are planned [8].

To this end, many different in-network caching strategies have been proposed in the literature, each adopting different perspectives and approaches in regard to the cooperation between caches, the criterion to cache a certain content or the network topology over which such caches are deployed. The performance of content-oriented networking has been studied under different application scenarios, with recent yet scarce contributions analyzing the performance gains that an information-centric network operation yields in vehicular environments [911] and in general, mobility-based adhoc networks [12]. However, most previous studies rely on very simplistic mobility assumptions such as a Manhattan grid as the road layout and constant vehicle speeds, which oversimplify the network model and do not capture properly the short-lived, spotty nature of the connectivity in such a communication environment.

In this paper we analyze how multi-objective heuristics can be applied to the allocation of cache profiles through the network on the assumption that travel patterns of the vehicles demanding such contents have been inferred a priori by predictive models. We find our motivation to address content caches at a profile level in the dynamic essence of the vehicular scenario under analysis, which makes it difficult to learn or predict user's profiles in a file basis. In particular our work will assess the Pareto trade-off between the utility of the cache allocation strategy and its economical cost. On one hand, a quantitative measure of the former optimization objective will be formulated as a function of the coverage of vehicles, the number of vehicles demanding contents from cached profiles and the effective duration of the caching session delivered to the users under coverage. On the other hand, the economical cost of the cache deployment will vary depending on the cached profile and the level of service - in terms of number of simultaneously served users. Once the problem has been formulated we will resort to multi-objective heuristics in order to determine the set of Paretooptimal caching strategies (namely, which profiles should be cached and served in the scenario) differently balancing the aforementioned goals. The performance of the proposed approach is discussed based on simulation results obtained from a realistic vehicular environment deployed over the city of Cologne, Germany.

The rest of the manuscript is structured as follows: first Section 2 will pose the notation and formulate the problem studied in this research work. Next, Sec- 
tion 3 describes the heuristic solver designed to tackle the optimization problem at hand. Section 4 presents and discusses the obtained simulation results and, finally, Section 5 concludes the paper and describes several future research lines of interest within the scope of this work.

\section{Problem Formulation}

According to the schematic diagram shown in Figure 1, we assume an urban scenario $S$ with $N$ nodes $\left\{v_{n}\right\}_{n=1}^{N}$ representing vehicles with V2I (Vehicular to Infrastructure) communication capabilities. It is assumed that a priori probabilistic knowledge about the routes of the vehicles has been acquired by virtue of soft-output predictive models (e.g. [13,14] and references therein), which can be modeled as a spatial-temporal probability density distribution $f_{t}^{n}(X, Y)$ such that $(X, Y)$ denotes geographical coordinates (e.g. latitude and longitude) and $\sum_{(x, y) \in S} f_{t}^{n}(X, Y)=1 \forall n \in\{1, \ldots, N\}$ and $\forall t \in\left[T_{\text {in }}^{n}, T_{\text {out }}^{n}\right]$. Here $T_{\text {in }}^{n}$ and $T_{\text {out }}^{n}$ stand for the time instants in which vehicle $v_{n}$ enters and leaves the scenario, respectively. The coordinates for a given vehicle $v_{n}$ at time will be given by $x_{n}(t)$ and $y_{n}(t)$. A circular coverage model with radii $r_{n}$ will be adopted.

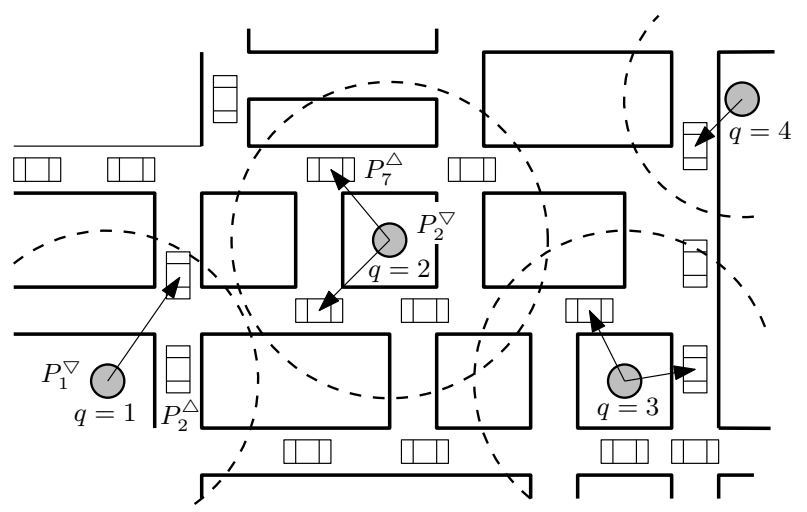

Fig. 1. Schematic diagram of the system model under consideration with $Q=4$ caches distributed over the scenario, $N=17$ vehicles and two cases highlighted: $P_{1}^{\nabla} \neq P_{2}^{\triangle}$ (i.e. content stored at cache $q=1$ is not that demanded by vehicle $n=2$ ) and $P_{2}^{\nabla}=P_{7}^{\triangle}$ (content stored at cache $q=2$ coincides with that requested by vehicle $n=7$ ).

The occupant at vehicle $v_{n}$ is subscribed to a certain content profile $P_{n}^{\triangle}$ from an $M$-sized portfolio $\mathcal{P} \triangleq\left\{\mathcal{P}_{1}, \ldots, \mathcal{P}_{M}\right\}$ designed and offered by the Internet Service Provider (ISP) at hand. These offered profiles are characterized by different resource levels demanded from the provider which, for the sake of simplicity, will be defined in terms of their required download rate $R_{m}[\mathrm{Mb} / \mathrm{sec}]$. For instance, profiles corresponding to high-definition video content will feature high values of $R_{m}$, whereas other services such as news feeds and blogs might correspond to content profiles with low values of $R_{m}$. Furthermore, it is assumed that each profile requires a minimum session establishment time $T_{m}^{e s t}$ [sec], such 
that the delivery of cached content is effective only after $T_{m}^{e s t}$ seconds since the vehicle demanding it entered the coverage area of the network cache from which the cached content is retrieved. In regards to the network cache we will assume $Q$ locations $\left\{\left(x_{q}, y_{q}\right)\right\}_{q=1}$ over the scenario $S$ at hand, each with coverage radii $r_{q}[\mathrm{~m}]$ such that communication between vehicle $n$ and cache $q$ can be possible if the distance between both extremes at time $t$ fulfills

$$
d_{n, q}^{t} \triangleq \sqrt{\left(x_{n}(t)-x_{q}\right)^{2}+\left(y_{n}(t)-y_{q}\right)^{2}}<\min \left\{r_{n}, r_{q}\right\} .
$$

Due to the need for data storage over network caches a cost of the overall caching strategy will be modeled as a simple, linear cost per cached unit of data $C_{\text {unit }}[$ monetary units/Mb]. By hereafter denoting the cached content at location $q \in\{1, \ldots, Q\}$ as $P_{q}^{\nabla} \in \mathcal{P} \bigcup\{\emptyset\}$ - with $\emptyset$ corresponding to the case when no content is cached at location $q$, yielding no associated cost -, the overall cost $\Phi\left(\mathbf{P}^{\nabla}\right)$ of the caching strategy $\mathbf{P} \nabla \triangleq\left\{P_{q}^{\nabla}\right\}_{q=1}^{Q}$ will be given by

$$
\Phi\left(\mathbf{P}^{\nabla}, T\right) \triangleq \sum_{q=1}^{Q} T R_{P_{q}^{\nabla}} C_{u n i t}, \text { [monetary units] }
$$

which will pose the first optimization goal of the problem tackled in this work. The second objective function will evaluate the inherent utility of the caching strategy $\mathbf{P} \nabla$ as a function of several factors: 1) the rate of the cached profile, whose contribution to the overall utility should be higher as more data are offloaded from the mobile network; 2) the matching between the content profile of the vehicles within coverage of every network cache in the scenario and the profile cached therein; and 3) the effective session duration, which is determined by the overall time that every vehicle is within reach of the network cache penalized by the session establishment time of the cached profile to be retrieved. Mathematically, this utility $U(\mathbf{P} \nabla, T)$ can be quantified as

$$
U\left(\mathbf{P}^{\nabla}, T\right) \triangleq \sum_{q=1}^{Q} R_{P_{q}^{\nabla}} \sum_{n=1}^{N} I\left(P_{n}^{\triangle}=P_{m}^{\nabla}\right)\left(T_{n, q}^{\varangle}-N_{n, q}^{\varangle} T_{P_{q} \nabla}^{e s t}\right),
$$

where $T_{n, q}^{\varangle}$ denotes the total time during which vehicle $n$ is within coverage of cache $q$, i.e.

$$
T_{n, q}^{\varangle} \triangleq \sum_{t=1}^{T} I\left(d_{n, q}^{t}<\min \left\{r_{n}, r_{q}\right\}\right),
$$

and $N_{n, q}^{\varangle}$ represents the number of total encounters between cache $q$ and vehicle $n$, which can be quantified by

$$
N_{n, q}^{\varangle} \triangleq \sum_{t=1}^{T} I\left(d_{n, q}^{t} \geq \min \left\{r_{n}, r_{q}\right\}\right) I\left(d_{n, q}^{t+1}<\min \left\{r_{n}, r_{q}\right\}\right),
$$

with $I(\cdot)$ in the above formulae being an auxiliary indicator function taking value 1 if its argument is true (and 0 otherwise). By including the soft estimations 
of the route followed by each vehicle in the network represented by the spacetemporal probability density function $f_{t}^{n}(X, Y)$, one obtains an estimation of the expected utility of the caching strategy $\mathbf{P} \nabla$ as $\mathbb{E}\{U(\mathbf{P} \nabla, T)\}$, where expectation is taken over the aforementioned distribution of the vehicles' coordinates.

Based on the above notation the optimization problem undertaken in this work can be conceived as the discovery of a $K$-sized set of caching strategies $\left\{\mathbf{P}_{k}^{\nabla, *}\right\}$ that optimally balances - in the Pareto sense of optimality - both their overall cost and utility over scenario $S$ and a time horizon of duration $T$. Mathematically,

$$
\left\{\mathbf{P}_{k}^{\nabla, *}\right\}_{k=1}^{K}=\underset{\mathbf{P} \nabla}{\arg } \max \mathbb{E}\left\{U\left(\mathbf{P}^{\nabla}, T\right)\right\}, \min \Phi\left(\mathbf{P}^{\nabla}, T\right),
$$

where the cardinality of the search space is given by $|\mathcal{P} \bigcup\{\emptyset\}|^{Q}=(M+1)^{Q}$, i.e. exponential with the number of network caches deployed and content profiles defined by the service provider. In other words, the goal is to determine which content profiles should be stored and served locally at caches $q \in\{1, \ldots, Q\}$ such that the expected utility of these cached profiles for the scenario at hand is maximum at a given cost level. Alternatively, $\left\{\mathbf{P}_{k}^{\nabla, *}\right\}_{k=1}^{K}$ represent the caching strategies with minimal cost at different levels of expected utility. As explained in the next section, the exponential search space from which solutions to the above problem are drawn will be efficiently explored by a bi-objective HS solver.

\section{Proposed Algorithm}

The bi-objective network caching problem posed in Expression (6) can be efficiently approached using the Harmony Search (HS) algorithm, a meta-heuristic solver first proposed in [15] and applied thereafter to a plethora of optimization problems arising in diverse disciplines such as Energy [16-18], Telecommunications [19,20], Manufacturing [21,22] and Data Mining [23, 24], among many others [25]. The search heuristic embodied in the HS algorithm is inspired by the music improvisation procedure and the progressive enhancement of the improvised melodies by musicians when pursuing aesthetically pleasant harmonies. From a computational standpoint the algorithm relies on a numerical solution encoding strategy, on which a set of operators is defined in a similar way to those featured by other meta-heuristic techniques from Evolutionary Computation and Swarm Intelligence [26]. In particular HS maintains a $K$-sized pool of candidate solutions or harmonies which are iteratively pushed towards regions of potentially higher optimality by virtue of the aforementioned operators. These operators emulate the memory-based and random pitch criteria by which musicians in practice vary their played notes (namely, optimization variables) until a stop criteria is met, e.g. a maximum number of improvisations $\mathcal{I}$ is reached.

For the problem at hand harmonies will encode a possible caching strategy as a $Q$-sized vector of integer numbers $\left\{\left\{X_{q}^{k}\right\}_{q=1}^{Q}\right\}_{k=1}^{K}$ such that $X_{q}^{k} \in\{0,1, \ldots, M\}$ correspond to the index of the cache profile proposed to be stored at cache $q \in$ $\{1, \ldots, Q\}$ in harmony $k \in\{1, \ldots, K\}$. A null value (i.e. $X_{q}^{k}=0$ ) reflects the case 
where no profile is allocated to the cache. Profile indices are sorted in increasing order of their bandwidth requirements $R_{m}$, sorting of utmost relevance for the effectiveness of the vicinity-based PAR operator explained in what follows. In regards to the HS flow diagram depicted in Figure 2 three are the operators that are applied to the harmonies contained within the memory for each iteration:

- Harmony Memory Considering Rate, controlled by a probabilistic parameter HMCR $\in[0,1]$ that sets the likelihood that the newly improvised value for a given note $X_{q}^{k}$ is drawn from the values $\left\{X_{q}^{1}, \ldots, X_{q}^{k-1}, X_{q}^{k+1}, \ldots, X_{q}^{K}\right\}$ taken by the same note in the other $K-1$ harmonies in the memory.

- Pitch Adjusting Rate, driven by the probability PAR $\in[0,1]$, establishes the probability that the value of a given note $X_{q}^{k}$ is replaced with any of its neighboring values in its corresponding alphabet $\{0,1, \ldots, M\}$, i.e.

$$
X_{q}^{k} \leftarrow \begin{cases}\max \left\{X_{q}^{k}-Z, 0\right\} & \text { with probability 0.5PAR, } \\ \min \left\{X_{q}^{k}+Z, M\right\} & \text { with probability 0.5PAR, } \\ X_{q}^{k} & \text { with probability 1-PAR, }\end{cases}
$$

where $Z$ is the realization of a uniform discrete random variable with support $\mathbb{N}[1, \lambda]$ (with $\lambda$ denoting bandwidth).

- Random Selection Rate, whose controlling parameter RSR $\in[0,1]$ sets the probability that the new value for a given note $X_{q}^{k}$ will be drawn uniformly at random (i.e. without any neighborhood consideration) from its corresponding alphabet. This is equivalent to the PAR process with $Z$ uniformly distributed over $\mathbb{Z}\left[-X_{q}^{k}, M-X_{q}^{k}\right]$

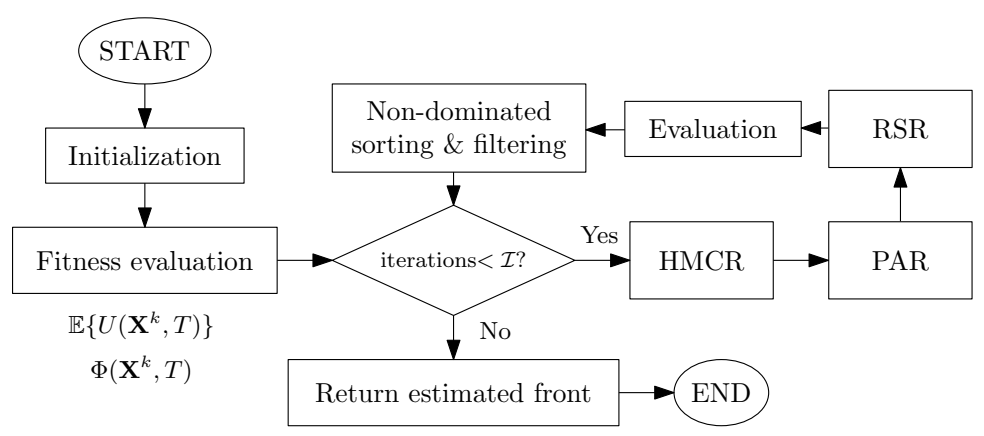

Fig. 2. Flow diagram of the proposed meta-heuristic solver.

These operators are sequentially applied to each note of the candidate solutions stored in the pool of harmonies. Once applied over the entire set of notes, the fitness functions defining the bi-objective caching criteria formulated in Section 2 - namely, $\mathbb{E}\left\{U\left(\mathbf{X}^{k}, T\right)\right\}$ and $\Phi\left(\mathbf{X}^{k}, T\right)$, with $\mathbf{X}^{k} \triangleq\left\{X_{1}^{k}, \ldots, X_{Q}^{k}\right\} \equiv \mathbf{P} \nabla$ - of the newly improvised solutions are evaluated. Based on the values of the objective functions for both the new harmonies and those from the previous iteration the entire set of $2 K$ harmonies are ordered and filtered following the 
well-known Pareto dominance ranking and crowding distance criterion. To be concise, each solution is assigned a numerical score equal to its dominance level (namely, 1 for the best, non-dominated subset of solutions, 2 for the second best level, and so forth). Once all solutions have been ranked, a second criterion hinging on the sum of distances to the closest harmony along each metric drives the ordering among the solutions within a certain dominance level: as such, those solutions featuring large crowding distances are preferred rather than those with small distances to their neighboring individuals in the Pareto space. Once this second ordering has been performed only the best $K$ solutions are kept in the pool of harmonies for the next iteration.

\section{Experiments and Results}

In order to assess the performance of the bi-objective heuristic solver explained in the previous section a vehicular scenario comprising realistic mobility traces over the city of Cologne (Germany) has been considered. A total of $N=13682$ vehicles are deployed in this simulation scenario, which depart from and arrive at different spots of the city during a time horizon of $T=3600$ seconds (from 8:00 to 9:00 AM). In order to shed more realism over the simulation the performed experiment utilizes the set of mobility traces released by TAPAS-Cologne [27], an initiative of the German Aerospace Center (ITS-DLR) to realistically reproduce urban vehicular traffic in this city. Caches are assumed to be located at the $Q=247$ cellular base stations deployed in the city, which have been retrieved from public German databases as of 2012, and made available by the same initiative. Coverage radii are set to $r_{n}=200$ and $r_{q}=1000$ meters for all vehicles and base stations, respectively. As for the cached content $M=5$ profiles will be considered to model diverse cached services by means of different bandwidths $\left\{R_{m}\right\}_{m=1}^{5}=\{0.01,0.1,0.2,0.5,1\}[\mathrm{Mb} / \mathrm{sec}]$. The unitary cost per cached $\mathrm{Mb}$ is set to $C_{\text {unit }}=100$ monetary units. The minimum session time for the content profiles will be given by $\left\{T_{m}^{e s t}\right\}_{m=1}^{M}=\{0,0.05,0.1,0.15,0.25\}$ [sec]. Without loss of generality a perfect estimation of the route followed by each vehicle will be assumed to reduce the computational complexity of the optimization algorithm: in this regard it should be noted that when dealing with soft estimations of the route followed by a car the caching optimization procedure would be identical, the difference being that the utility would require averaging over both space and time domains as per (6). The HS solver is configured with a pool of $K=100$ harmonies, $\mathrm{HMCR}=0.7, \mathrm{PAR}=\mathrm{RSR}=0.1, \lambda=1$ and a total of $\mathcal{I}=200$ iterations. These parameters have been tuned via offline simulations over a value grid (not shown due to lack of space).

The obtained results are summarized in Figures 3.a through 3.d, which depict the estimated Pareto front by the proposed HS solver along with three different solutions differently balancing the optimization criteria: utility and cost of the deployment. As we can observe, the caching strategies highlighted in the Pareto front span from the minimum-utility, minimum-cost region to solutions favoring high utility values at higher cost penalties. Intuitively one should expect that 
the former corresponds to a caching strategy where a large fraction of the caches located at the base stations do not store any content profile (especially those in the outskirts of the city to minimize the impact of the lack of caches), whereas the latter should feature a relatively higher number of high-rate cached profiles, mostly located in the city center where more vehicular traffic occurs.

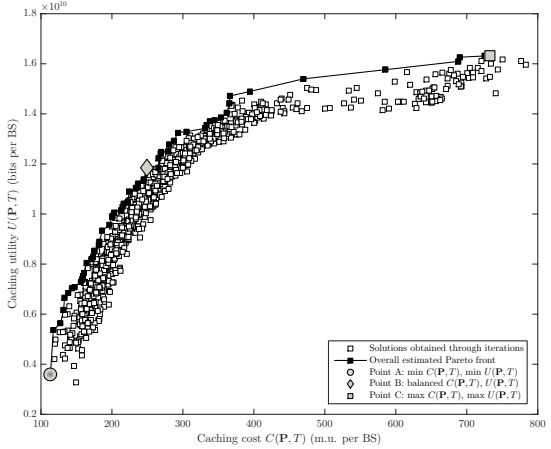

(a)

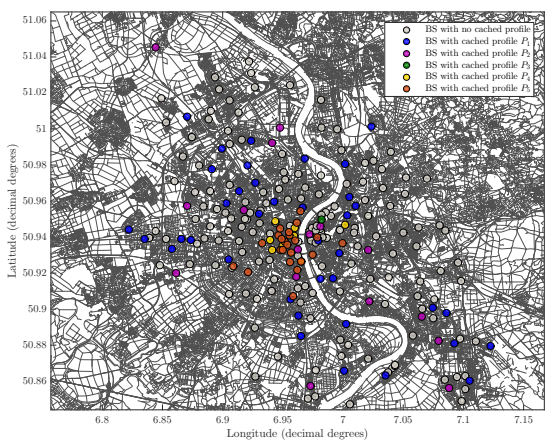

(c)

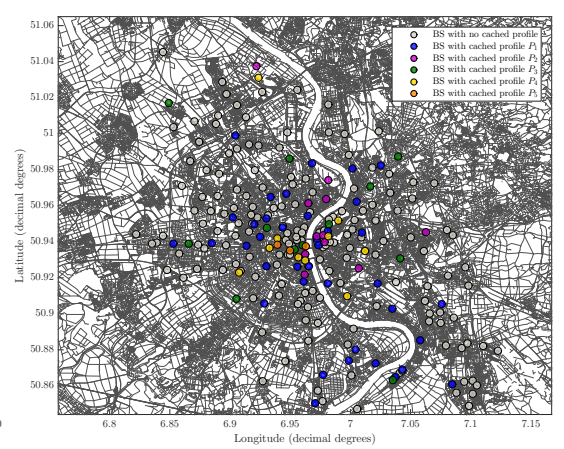

(b)

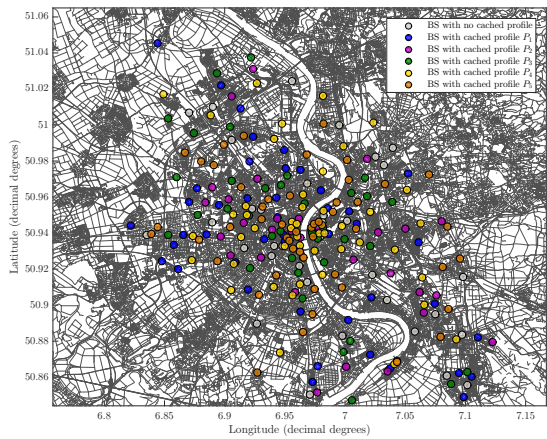

(d)

Fig. 3. (a) Estimated front by the bi-objective HS solver after $\mathcal{I}=200$ iterations; (b) Network cache (solution) of point A in the estimated front; (c) point B; (d) point C.

This prior intuition gets confirmed through the obtained plots. To begin with, the cache deployment in Figure 3.b corresponds to Point A, namely, an strategy with minimum cost and consequently the lowest utility along the estimated Pareto front. It can be noticed that the deployment sets no contents in most caches through the urban scenario, while those activated by the caching solution concentrate around the city center and serve contents with generally low rate demands. As the strategy becomes less restrictive in terms of costs not only the set of cached contents is larger in terms of cardinality (i.e. more network caches with stored profiles are included in the strategy), but also are such strategies more diverse and sparsely distributed over the city, as Figure 3.c clearly shows for an intermediate solution in the Pareto front (Point B). Finally, Figure 3.d depicts the deployed caches associated to Point C, i.e. an strategy with no cost requirements and a maximal utility for the demands of the deployed vehicles. It 
can be observed that in this third example empty caches are kept to a minimum, with a large concentration of high-rate content profiles cached over the scenario.

\section{Concluding Remarks and Future Research Lines}

This work has capitalized on the paramount need for efficient mechanisms to optimally allocate network caches in urban environments so as to keep data traffic redundantly retrieved from nearby locations to their minimum. In this regard a novel formulation of the cost-efficient deployment of network caches over mobility-based scenarios has been proposed, which models both the coupling between the demanded content from the vehicle and the served profile by the network cache, but also the delay penalty due to the session establishment of the service at hand and the cost associated to the storage of the requested content. To efficiently solve the formulated problem in a centralized manner a heuristic technique based on a bi-objective version of the HS algorithm has been designed and put to practice over a realistic urban scenario. The Pareto-optimal caching strategies estimated by the proposed heuristic method meet the intuition and span a wide portfolio of caching options, which pave the way towards new formulations of this problem accounting for finite capacity constraints at the caches and/or mobile caches enabled at the vehicles.

\section{Acknowledgments}

This work has been supported by the Basque Government through the ELKARTEK program (ref. KK-2015/0000080) and the BID3ABI project.

\section{References}

1. Cisco: Visual Networking Index: Forecast and Methodology 2013-2018. White Paper. Available: http://www.cisco.com/go/vni (2014)

2. Luotonen, Ari: Web Proxy Servers. Prentice Hall (1997)

3. Wessels, Duane: Web Caching. O'Reilly and Associates (2001)

4. Wang, X., Chen, M., Taleb, T., Ksentini, A., Leung, V. C.: Cache in the Air: Exploiting Content Caching and Delivery Techniques for 5G Systems. IEEE Communications Magazine 52(2), 131-139 (2014)

5. ElBamby, M. S., Bennis, M., Saad, W., Latva-Aho, M.: Content-aware User Clustering and Caching in Wireless Small Cell Networks. IEEE International Symposium on Wireless Communications Systems, 945-949 (2014)

6. Syed, T. Bennis, M., Nardelli, P., Latva-aho, M.: Caching in Wireless Small Cell Networks: A Storage-Bandwidth Trade-off. IEEE Comm. Letters, to appear (2016)

7. Ahlgren, B., Dannewitz, C., Imbrenda, C., Kutscher, D., Ohlman, B.: A Survey of Information-Centric Networking. IEEE Comm. Mag. 50(7), 26-36 (2012)

8. Xylomenos, G., Ververidis, C., Siris, V., Fotiou, N., Tsilopoulos, C., Vasilakos, X., Katsaros, K., Polyzos, G.: A Survey of Information-Centric Networking Research. IEEE Communication Surveys and Tutorials 16(2), 1024-1049 (2013) 
9. Amadeo, M., Campolo, C., Molinaro, A.: CRoWN: content-centric networking in vehicular ad hoc networks. IEEE Communications Letters, 16(9), 1380-1383 (2012)

10. Amadeo, M., Campolo, C., Molinaro, A. Enhancing content-centric networking for vehicular environments. Computer Networks, 57(16), 3222-3234 (2013)

11. TalebiFard, P., Leung, V. C., Amadeo, M., Campolo, C., Molinaro, A. InformationCentric Networking for VANETs. Vehicular ad hoc Networks, 503-524 (2015)

12. Liu, X., Li, Z., Yang, P., Dong, Y. Information-Centric Mobile Ad Hoc Networks and Content Routing: A Survey. Ad Hoc Networks (2016).

13. Xue, G., Li, Z., Zhu, H., Liu, Y.: Traffic-known Urban Vehicular Route Prediction based on Partial Mobility Patterns. International Conference on Parallel and Distributed Systems, 369-375 (2009)

14. Chen, L., Lv, M., Ye, Q., Chen, G., Woodward, J.: A Personal Route Prediction System based on Trajectory Data Mining. Inf. Sci. 181(7), 1264-1284 (2011)

15. Geem, Z. W., Kim, J.-H., Loganathan, G. V.: A New Heuristic Optimization Algorithm: Harmony Search. Simulation 76(2), 60-68 (2001)

16. Ceylan, H., Ceylan, H., Haldenbilen, S., Baskan, O.: Transport Energy Modeling with Meta-heuristic Harmony Search Algorithm: an Application to Turkey. Energy Policy 36(7), 2527-2535 (2008)

17. Vasebi, A., Fesanghary, M., Bathaee, S. M. T.: Combined Heat and Power Economic Dispatch by Harmony Search Algorithm. International Journal of Electrical Power \& Energy Systems 29(10), 713-719 (2007)

18. Salcedo-Sanz, S., Pastor-Sanchez, A., Del Ser, J., Prieto, L., Geem, Z. W.: A Coral Reefs Optimization Algorithm with Harmony Search Operators for Accurate Wind Speed Prediction. Renewable Energy 75, 93-101 (2015)

19. Del Ser, J., Bilbao, M. N., Gil-Lopez, S., Matinmikko, M., Salcedo-Sanz, S.: Iterative Power and Subcarrier Allocation in Rate-Constrained Orthogonal Multicarrier Downlink Systems based on Hybrid Harmony Search Heuristics. Engineering Applications of Artificial Intelligence 24(5), 748-756 (2011)

20. Landa-Torres, I., Gil-Lopez, S., Del Ser, J., Salcedo-Sanz, S., Manjarres, D., Portilla-Figueras, J. A.: Efficient Citywide Planning of Open WiFi Access Networks using Novel Grouping Harmony Search Heuristics. Engineering Applications of Artificial Intelligence 26(3), 1124-1130 (2013)

21. Garcia-Santiago, C. A., Del Ser, J., Upton, C., Quilligan, F., Gil-Lopez, S., SalcedoSanz, S.: A Random-Key Encoded Harmony Search Approach for Energy-Efficient Production Scheduling with Shared Resources. Eng. Opt. 47(11), 1481-1496 (2015)

22. Pan, Q. K., Suganthan, P. N., Liang, J. J., Tasgetiren, M. F.: A Local-Best Harmony Search Algorithm with Dynamic Sub-Harmony Memories for Lot-Streaming Flow Shop Scheduling Problem. Expert Sys. with App. 38(4), 3252-3259 (2011)

23. Agustín-Blas, L.E., Salcedo-Sanz, S., Jiménez-Fernández, S., Carro-Calvo, L., Del Ser J., Portilla-Figueras, J.A.: A New Grouping Genetic Algorithm for Clustering Problems. Expert Systems with Applications 39(10), 9695-9703 (2012)

24. Karimi, Z., Abolhassani, H., Beigy, H.: A New Method of Mining Data Streams using Harmony Search. J. of Intelligent Information Systems 39(2), 491-511 (2012)

25. Manjarres, D., Landa-Torres, I., Gil-Lopez, S., Del Ser, J., Bilbao, M. N., SalcedoSanz, S., Geem, Z. W.: A Survey on Applications of the Harmony Search Algorithm. Engineering Applications of Artificial Intelligence 26(8), 1818-1831 (2013)

26. Engelbrecht, A. P.: Fundamentals of Computational Swarm Intelligence. John Wiley \& Sons (2006)

27. Uppoor, S., Trullols-Cruces, O., Fiore, M., Barcelo-Ordinas, J. M.: Generation and Analysis of a Large-Scale Urban Vehicular Mobility Dataset. IEEE Transactions on Mobile Computing 13(5), 1061-1075 (2014) 